\title{
Unsteady Free Convection Flow between Two Vertical Parallel Plates with Newtonian Heating
}

\author{
${ }^{1}$ Fasihah Zulkiflee*, ${ }^{2}$ Ahmad Qushairi Mohamad, ${ }^{3}$ Sharidan Shafie and ${ }^{4}$ Arshad Khan \\ $1,2,3$ Department of Mathematical Sciences, Universiti Teknologi Malaysia \\ 81310 UTM Johor Bahru, Malaysia \\ ${ }^{4}$ Department of Computer Science/IT, Sarhad University of Science \& IT \\ Peshawar Khyber Pakhthunkhwa, Pakistan \\ *Corresponding author: fasihahzulkiflee92@gmail.com
}

Article history

Received: 30 September 2018

Received in revised form: 28 March 2019

Accepted: 2 June 2019

Published online: 1 August 2019

\begin{abstract}
Free convection flow in a boundary layer region is a motion that results from the interaction of gravity with density differences within a fluid. These differences occur due to temperature or concentration gradients or due to their composition. Studies pertaining free convection flows of incompressible viscous fluids have received much attention in recent years both theoretically (exact or approximate solutions) and experimentally. The situation where the heat be transported to the convective fluid via a bounding surface having finite heat capacity is known as Newtonian heating (or conjugate convective flows). In this paper, the unsteady free convection flow of an incompressible viscous fluid between two parallel plates with Newtonian heating is studied. Appropriate nondimensional variables are used to reduce the dimensional governing equations along with imposed initial and boundary conditions into dimensionless forms. The exact solutions for velocity and temperature are obtained using the Laplace transform technique. The corresponding expressions for skin friction and Nusselt number are also calculated. The graphical results are displayed to illustrate the influence of various embedded parameters such as Newtonian heating parameter and Grashof number. The results show that the effect of Newtonian heating parameter increases the Nusselt number but reduces the skin friction.
\end{abstract}

Keywords Free convection; vertical parallel plate; Newtonian heating; Laplace transform.

Mathematics Subject Classification 76D05, 35Q35.

\section{Introduction}

The general area of natural or free convection in vertical parallel plate channels for a single phase has received a great deal of attention in previous years due to the fact that many practical applications involve natural convection heat transfer [1]. There has been a great interest and 
research activity in free convection in vertical parallel plate. Generally, the problems of free convection flows in parallel plate are usually modeled under the assumptions of constant surface temperature, ramped wall temperature, or constant surface heat flux [2-7]. Viscous dissipation effects on parallel plates with constant heat flux boundary conditions was investigated by Tso [8] while Paul et al. [6] investigate transient free convective flow in a vertical channel with constant temperature and constant heat flux on the walls. Kundu et al. [9] the effects the viscous dissipation between two asymmetrically heated infinitely long parallel plates while effects of wetted walls on laminar natural convection between vertical parallel plates with asymmetric heating was investigate by Tsay [10].

However, in many practical situations where the heat transfer from the surface is taken to be proportional to the local surface temperature, the above assumptions fail to work. Such types of flows are termed as conjugate convective flows, and the proportionally condition of the heat transfer to the local surface temperature is termed as Newtonian heating [11]. Lesnic et al. [11] investigated free convection boundary-layer flow above a nearly horizontal surface in a porous medium with Newtonian heating while Shehzad et al. [12] investigate three-dimensional flow of Jeffrey fluid with Newtonian heating. There are many researchers consider Newtonian heating in their research such as [13-18] but not many considered Newtonian heating in between two parallel plates. Akbar et al. [19] were discussing effect of Newtonian heating for mixed convective magnetohydrodynamics peristaltic flow of jeffrey fluid while Hamza [20] investigate the influence of Navier Slip and Newtonian heating in transient flow of an exothermic fluid in vertical channel. The main objective of the present paper is to investigate the influence of Newtonian heating in free convection flow in vertical parallel plate.

\section{Mathematical Analysis}

In the present study, an unsteady flow of a viscous incompressible electrical conducting fluid between two vertical plates with Newtonian heating is considered. The $x^{\prime}$-axis is taken along one of the plates in the vertically upward direction and the $y^{\prime}$-axis is taken normal to the plates. Initial, at time $t^{\prime} \leq 0$, the two plates and the fluid are assumed to be the same temperature $T_{h}^{\prime}$. At time $t^{\prime}>0$, the temperature of the plate at $y^{\prime}=0$ are raised to $T_{w}^{\prime}$ and causing the flow convection currents. Then the flow can be shown to be governed by the usual Boussinesq's approximations as presented below and Figure 1 shows the flow configuration and coordinates systems of the flow.

$$
\begin{gathered}
\frac{\partial u^{\prime}}{\partial t^{\prime}}=\nu \frac{\partial^{2} u^{\prime}}{\partial y^{\prime 2}}+g \beta\left(T^{\prime}-T_{h}^{\prime}\right) \\
\rho C_{p} \frac{\partial T^{\prime}}{\partial t^{\prime}}=k \frac{\partial^{2} T^{\prime}}{\partial y^{\prime 2}}
\end{gathered}
$$

The initial and boundary conditions of the problem are

$$
\begin{array}{ll}
t^{\prime} \leq 0: & u^{\prime}=0, T^{\prime}=T_{h}^{\prime} \text { for } 0 \leq y^{\prime} \leq h \\
t^{\prime}>0: & u^{\prime}=U_{0}, \frac{\partial T^{\prime}}{\partial y^{\prime}}=-\frac{h_{s}}{k} T^{\prime}, \text { at } y^{\prime}=0 \\
& u^{\prime}=0, T^{\prime}=T_{h}^{\prime}, \text { at } y^{\prime}=h
\end{array}
$$




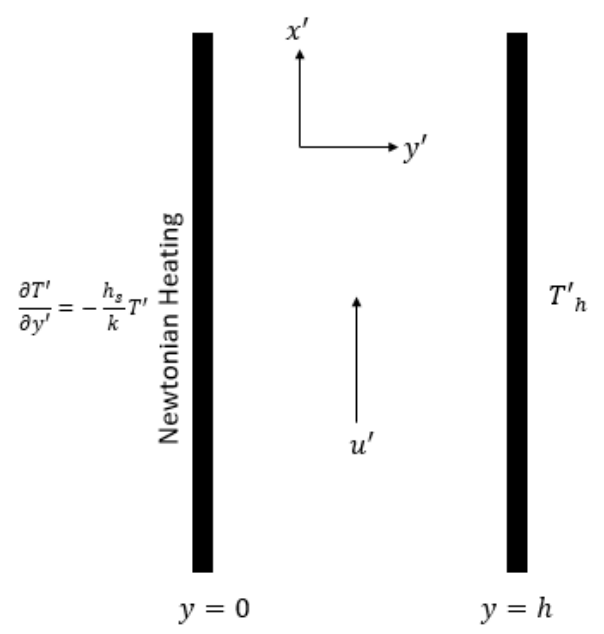

Figure 1: Flow configuration

Here $u^{\prime}$ is the velocity of the fluid, $g$ is the acceleration due to gravity, $\beta$ is volumetric coefficients of thermal expansion, $t^{\prime}$ is time, $h$ is the distance between two vertical plates, $T^{\prime}$ is the temperature of the fluid, $T_{h}^{\prime}$ is the temperature of the plate at $y^{\prime}=h, \nu$ is the kinematic viscosity, $k$ is the thermal conductivity of fluid, $T_{w}^{\prime}$ temperature of the plate at $y^{\prime}=0$ and $h_{s}$ is the heat transfer coefficient. The non-dimensional quantities are now introduced as

$$
\begin{aligned}
& y=\frac{y^{\prime} U_{0}}{\nu}, t=\frac{t^{\prime} U_{0}^{2}}{\nu}, u=\frac{u^{\prime}}{U_{0}}, \\
& \theta=\frac{T^{\prime}-T_{h}^{\prime}}{T_{w}^{\prime}-T_{h}^{\prime}}, \mu=\rho \nu, H=\frac{U_{0} h}{\nu},
\end{aligned}
$$

where $u$ is the non-dimensional velocity, $y$ is non-dimensional coordinates axis normal to the plates, $t$ is the non-dimensional time, $\theta$ is non-dimensional temperature, $\mu$ is the coefficient of viscosity and $H$ is the non-dimensional distance between the plates. Equation (1)-(3) with (4) will reduce the equations as

$$
\begin{gathered}
\frac{\partial u}{\partial t}=\frac{\partial^{2} u}{\partial y^{2}}+G r \theta \\
\operatorname{Pr} \frac{\partial \theta}{\partial t}=\frac{\partial^{2} \theta}{\partial y^{2}}
\end{gathered}
$$

The initial and boundary conditions are

$$
\begin{aligned}
t \leq 0: & u=0, \theta=0, \text { for } 0 \leq y \leq H, \\
t>0: & u=1, \frac{\partial \theta}{\partial t}=-\gamma(\theta+1), \text { at } y=0 \\
& u=0, \theta=0, \text { at } y=H,
\end{aligned}
$$


where $G r=\frac{\nu g \beta\left(T_{w}^{\prime}-T_{h}^{\prime}\right)}{U_{0}^{3}}$ is thermal Grashof number, $\operatorname{Pr}=\frac{\mu C_{p}}{k}$ is Prandtl number and $\gamma=\frac{h_{s} \nu}{U_{0} k}$ is the Newtonian heating parameter. The solutions of velocity and temperature after solving equations (5) and (6) with the initial and boundary conditions (7) by using Laplace transform technique are obtained as

$$
\begin{gathered}
u(y, t)=\sum_{n=0}^{\infty}\left[f_{1}(a, t)-f_{1}(b, t)\right]+ \\
\frac{a_{2}}{a_{1}} \sum_{n=0}^{\infty} \sum_{m=0}^{n}(-1)^{m}\left[f_{3}(c, t)-f_{3}(d, t)-f_{3}(g, t)+f_{3}(h, t)\right] \\
+\frac{a_{2}}{a_{1}} \sum_{n=0}^{\infty}(-1)^{n}\left[f_{3}(b \sqrt{P r}, t)-f_{3}(a \sqrt{P r}, t)\right] \\
\theta(y, t)=\sum_{n=0}^{\infty}(-1)^{n}\left[k_{1}\left[f_{2}(a \sqrt{P r}, t)-f_{2}(b \sqrt{P r}, t)\right]\right. \\
\left.+k_{2}\left[f_{2}(a \sqrt{P r}, t)-f_{2}(b \sqrt{P r}, t)\right]\right]
\end{gathered}
$$

where

$$
\begin{gathered}
a=2 n H+y \\
b=2 n H+2 H-y \\
c=2 m H \sqrt{P r}+a \\
d=2 m H \sqrt{P r}+b \\
g=2 m H \sqrt{\operatorname{Pr}}+2 H \sqrt{P r}+a \\
h=2 m H \sqrt{\operatorname{Pr}}+2 H \sqrt{\operatorname{Pr}}+b \\
k_{1}=\frac{\gamma}{\sqrt{P r}} \\
k_{2}=\frac{\gamma^{2} H}{\sqrt{P r}} \\
a_{1}=\operatorname{Pr}-1 \\
a_{2}=\frac{G r\left(\gamma+\gamma^{2} H\right)}{\sqrt{\operatorname{Pr}}} \\
f_{1}(z, t)=\operatorname{Erfc}\left(\frac{z}{2 \sqrt{t}}\right) \\
f_{2}(z, t)=2 \sqrt{\frac{t}{\pi}} e^{\frac{-z^{2}}{4 t}}-z E r f c\left(\frac{z}{2 \sqrt{t}}\right)
\end{gathered}
$$




$$
f_{3}(z, t)=\frac{1}{3}\left(4 t+z^{2}\right) \sqrt{\frac{t}{\pi}} e^{\frac{-z^{2}}{4 t}}-\left(z t+\frac{z^{3}}{6}\right) \operatorname{Erfc}\left(\frac{z}{2 \sqrt{t}}\right)
$$

Here, $z$ is a dummy variable and $f_{1}, f_{2}, f_{3}$ are functions of dummy variable. Using expressions (8) and (9), the skin friction and the rate of heat transfer are given by,

$$
\tau=\frac{\tau^{\prime} h}{\mu U}=-\left.\frac{\partial u}{\partial y}\right|_{y=0}
$$

or

$$
\begin{gathered}
\tau=\sum_{n=0}^{\infty}\left[f_{5}(r, t)+f_{5}(s, t)\right] \\
+\left(\frac{a_{2}}{a_{1}}\right)^{2} \sum_{n=0}^{\infty} \sum_{m=0}^{n}(-1)^{m}\left[f_{5}\left(r_{1}, t\right)-f_{5}\left(r_{2}, t\right)-f_{5}\left(r_{3}, t\right)+f_{5}\left(r_{4}, t\right)\right] \\
+\frac{a_{2}}{a_{1}} \sum_{n=0}^{\infty}(-1)^{n}\left[f_{6}\left(r_{1} \sqrt{P r}, t\right)-f_{6}\left(r_{2} \sqrt{P r}, t\right)\right]
\end{gathered}
$$

and

$$
N u=\left.\frac{q h}{k\left(T^{\prime}-T^{\prime} h\right)} \frac{\partial T^{\prime}}{\partial y^{\prime}}\right|_{y=0}=1+\frac{1}{\theta(0, t)}
$$

where

$$
\begin{gathered}
r_{1}=2 n H \\
r_{2}=2 n H+2 H \\
r_{3}=2 m H \sqrt{P r}+r \\
r_{4}=2 m H \sqrt{P r}+s \\
r_{5}=2 m H \sqrt{P r}+2 H \sqrt{P r}+r \\
r_{6}=2 m H \sqrt{P r}+2 H \sqrt{P r}+s \\
f_{4}(z, t)=\frac{e^{-\frac{z^{2}}{4 t}}}{\sqrt{\pi t}} \\
f_{5}(z, t)=\frac{2}{3} z \sqrt{\frac{t}{\pi}} e^{-\frac{z^{2}}{4 t}}+\frac{z\left(t+\frac{z^{2}}{6}\right)}{\sqrt{\pi t}} e^{-\frac{z^{2}}{4 t}}-\frac{z\left(4 t+z^{2}\right)}{6 \sqrt{\pi t}} e^{-\frac{z^{2}}{4 t}} \\
-\frac{1}{3} z^{2} \operatorname{Erfc}\left[\frac{z}{2 \sqrt{t}}\right]-\left(t+\frac{z^{2}}{6}\right) \operatorname{Erfc}\left[\frac{z}{2 \sqrt{t}}\right]
\end{gathered}
$$




$$
\begin{gathered}
f_{6}(z, t)=-\frac{2 \operatorname{Pr} z \sqrt{t}}{3 \sqrt{\pi}} e^{-\frac{P r z^{2}}{4 t}}-\frac{\sqrt{\operatorname{Pr}} z\left(t+\frac{\operatorname{Pr} z^{2}}{6}\right) \sqrt{t}}{3 \sqrt{\pi}} e^{-\frac{P r z^{2}}{4 t}}+\frac{\operatorname{Pr}^{\frac{3}{2}} z^{2}}{3} \operatorname{Erfc}\left[\frac{z}{2 \sqrt{t}}\right] \\
+\frac{\operatorname{Pr} z\left(4 t+\operatorname{Pr} z^{2}\right)}{6 \sqrt{\pi t}} e^{-\frac{\operatorname{Pr} z^{2}}{4 t}}+\sqrt{\operatorname{Pr}}\left(t+\frac{\operatorname{Pr} z^{2}}{6}\right) \operatorname{Erfc}\left[\frac{z}{2 \sqrt{t}}\right] \\
\theta(0, t)=\sum_{n=0}^{\infty}(-1)^{n}\left[k_{1}\left[f_{2}(2 n H \sqrt{\operatorname{Pr}}, t)-f_{2}(2 H(n+1) \sqrt{\operatorname{Pr}}, t)\right]\right. \\
\left.+k_{2}\left[f_{2}(2 n H \sqrt{\operatorname{Pr}}, t)-f_{2}(2 H(n+1) \sqrt{\operatorname{Pr}}, t)\right]\right]
\end{gathered}
$$

\section{Results and Discussion}

In order to get the physical insight of the problem, the numerical values of the temperature field, velocity field, skin friction and Nusselt number are computed for different values of the parameters such as Newtonian heating parameter, Grashof number, Prandtl number and time.The effects of these parameters on velocity and temperature fields are depicted in Figures 2-10.

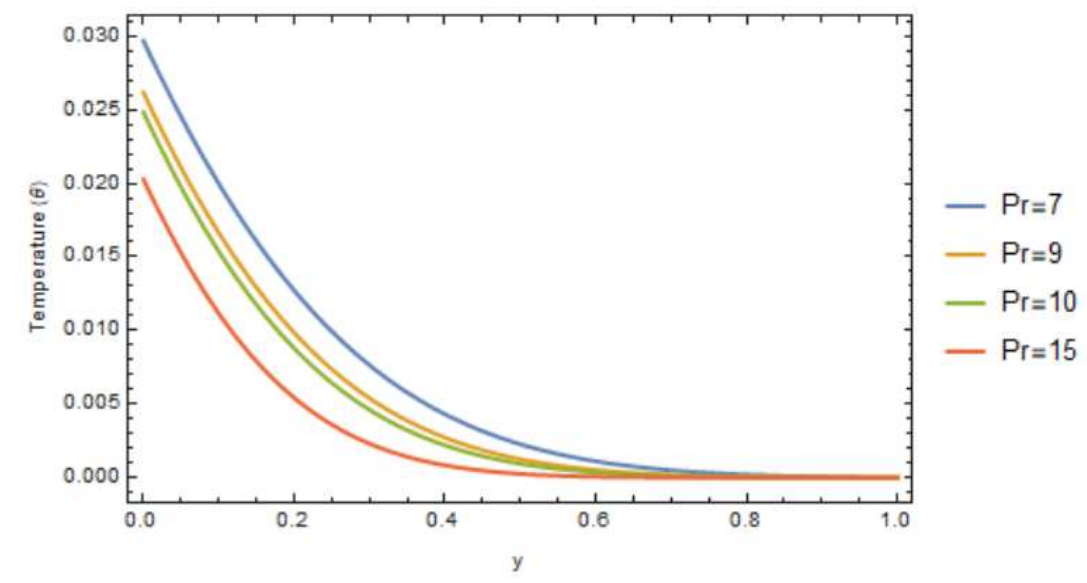

Figure 2: Temperature Profiles with Different $\operatorname{Pr}$ with $\gamma=0.1, H=1, t=0.4$

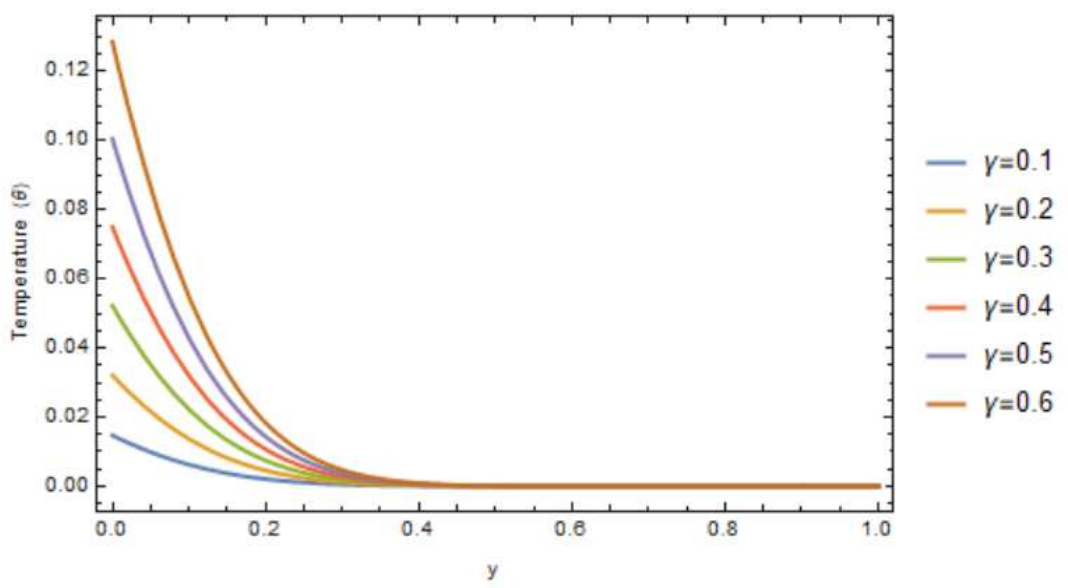

Figure 3: Temperature Profiles with Different $\gamma$ for $\operatorname{Pr}=0.71, H=1, t=0.1$ 
Figures 2 and 4 present the temperature profiles which include different values of $\operatorname{Pr}$ number and different values of t. It is found when the $\operatorname{Pr}$ number inreases, the temperature profiles will decrease. This is because for small values of $\operatorname{Pr}$ number, the fluid in highly thermal conductive. Physically, if $\operatorname{Pr}$ increases, the thermal diffusivity decreases and these phenomena lead to the decreasing of energy ability that reduces the thermal boundary layer. Figure 3 shows the effect of $\gamma$ in the temperature. It is seen that increasing the $\gamma$ will cause increase in temperature.

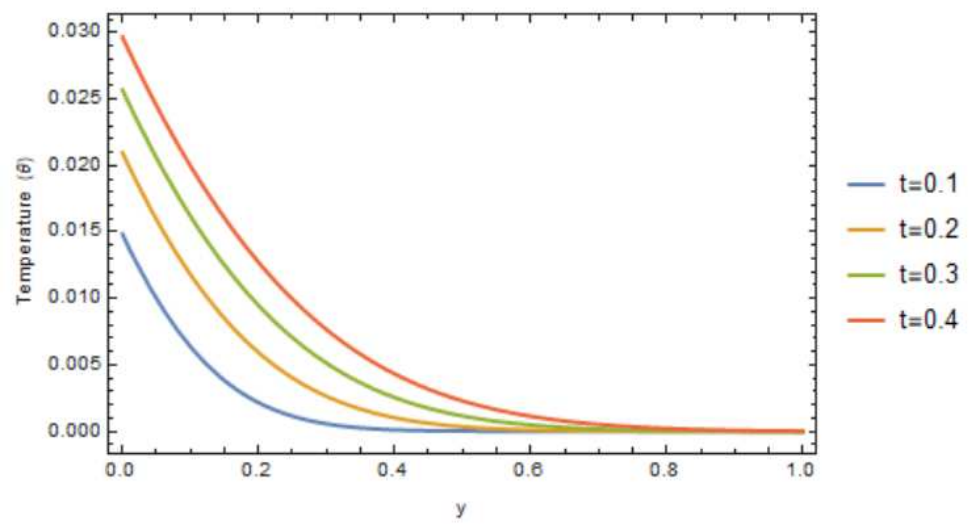

Figure 4: Temperature Profiles with Different Values of $t$ with $\operatorname{Pr}=7, \gamma=0.1, H=1, t=0.1$

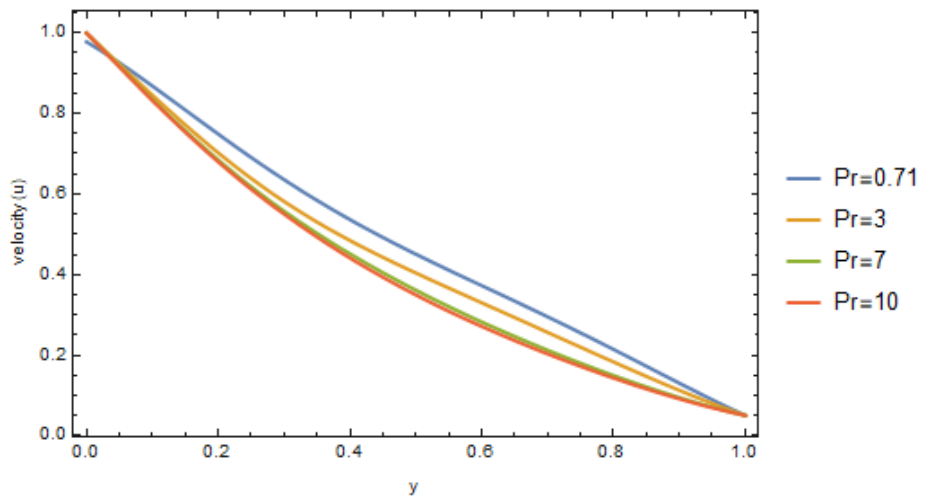

Figure 5: Velocity Profiles with Different $\operatorname{Pr}$ and $\gamma=0.1, H=1, t=0.4$

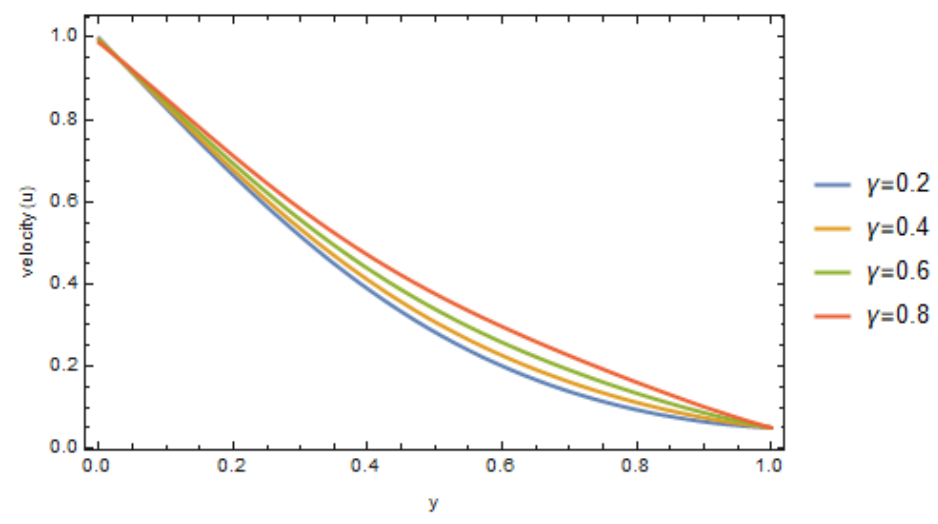

Figure 6: Velocity Profiles with Different $\gamma$ for $\operatorname{Pr}=0.71, G r=5, H=1, t=0.1$. 
Velocity profiles graph are presented in Figures 5-7. Figure 5 shows velocity profiles with different $\operatorname{Pr}$. It is seen that increasing the value of $\operatorname{Pr}$ will decrease the velocity of the fluid. Physically this is possible because fluid with higher Prandtl number have greater viscosity which makes the fluid thick and hence move slowly. While Figure 6 shows the velocity profiles with different $\gamma$ and Figure 7 shows velocity profiles with different values of t. Increasing the $\gamma$ number and $t$ will increase the velocity of the fluid.

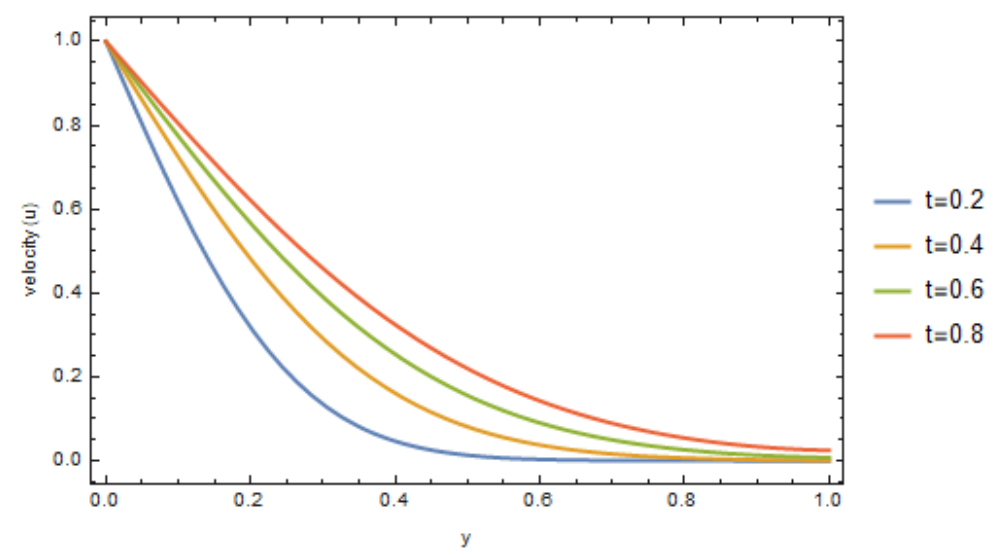

Figure 7: Velocity Profiles with Different Values of $t$ for $\gamma=0.1 \operatorname{Pr}=0.71, G r=5, H=1$.

In Figures 8 - 10 each of them illustrates the variation of skin friction and Nusselt number against time, Figures 8 and 9 show skin friction against $t$ for different $G r$ number. The effect of increasing $G r$ number is to decrease the shearing stress on the surface. It is also noticed that as the time passes, the skin friction rises for $G r<0$ while it falls for $G r>0$. For Figure 10, it is observed that increasing $\operatorname{Pr}$ number enhances the heat transfer coefficient. The positive values of Nusselt number show that the heat is transferred from the surface to the medium, which results in positive heat transfer coefficient. Further, it is noticed that the increase in $t$ leads to a decrease in Nusselt number.

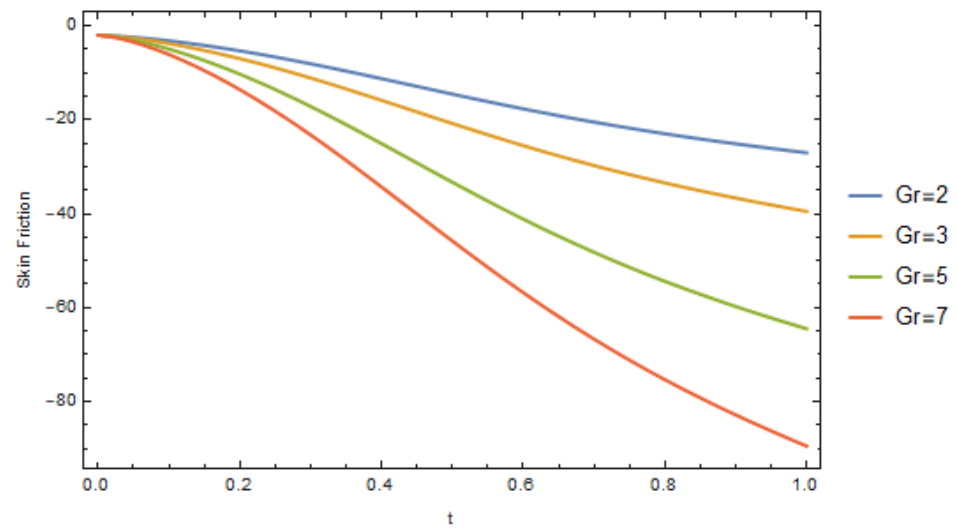

Figure 8: Skin Friction against $\mathrm{t}$ for $G r>0$ and $\gamma=2, H=1, \operatorname{Pr}=0.71$ 


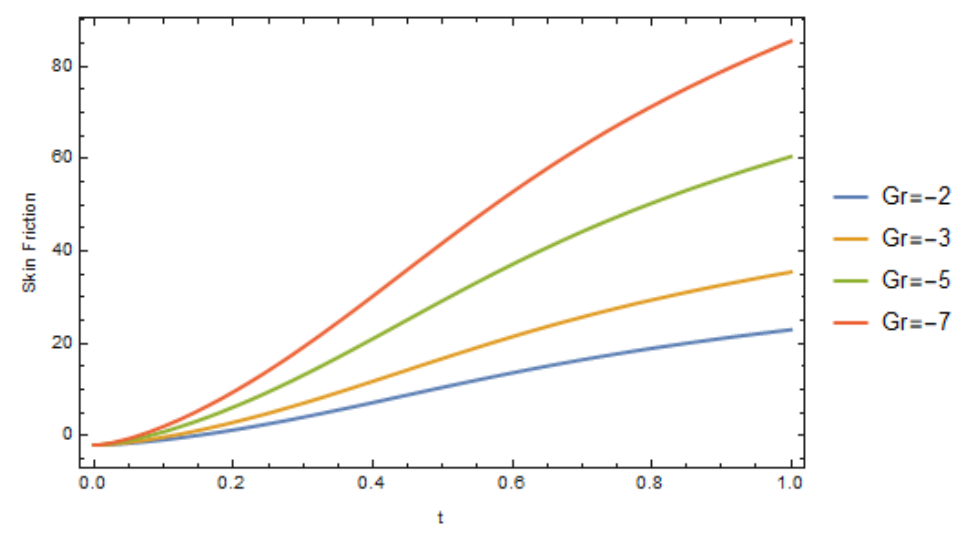

Figure 9: Skin Friction against $t$ for $G r<0$ and $\gamma=2, H=1, \operatorname{Pr}=0.71$

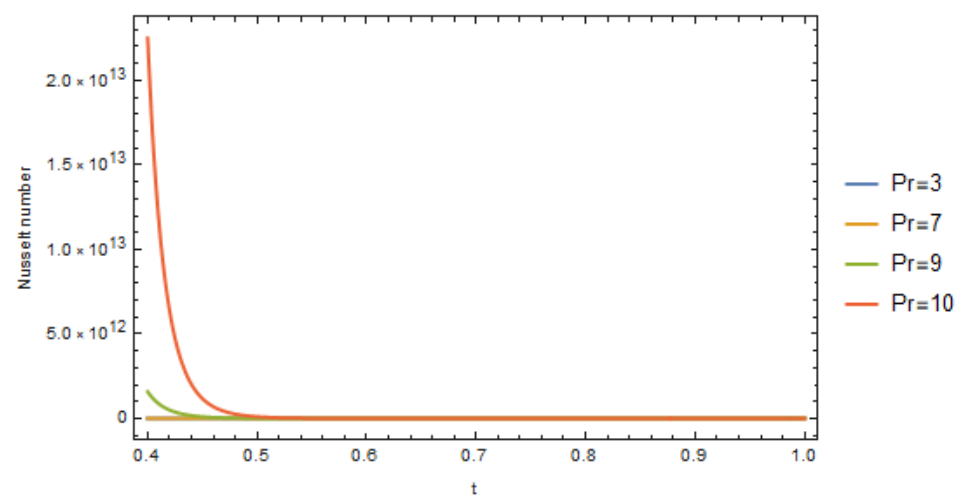

Figure 10: Nusselt Number against $t$ for $\gamma=0.5, H=1$

\section{Conclusion}

Free convection boundary layer flow between two vertical parallel plates with Newtonian heating have been studied theoretically. The dimensionless governing linear partial differential equations are solved analytically using Laplace transfom method. Numerical calculations have been calculated from different parameter such as Newtonian heating parameter $\gamma$, Grashof number Gr and Prandtl number Pr. A brief summary of the major findings are as follows:

1. Fluid velocity decreases with an increase in the Prandtl number and increases with increasing the Grashof number.

2. The fluid temperature decreases with an increase of Prandtl number.

3. The velocity and temperature of the fluid are enhanced with an increase in the Newtonian heating parameter.

4. The skin friction is decreasing with increasing the Grashof number.

5. Nusselt number increase when Prandtl number is increasing.

6. The solutions satisfied the given boundary conditions. 


\section{Acknowledgments}

The authors would like to acknowledge UTM Zamalah, Ministry of Higher Education (MOHE) and Research Management Centre-UTM, Universiti Teknologi Malaysia (UTM) for the financial support through vote number 5F004 for this research.

\section{References}

[1] Al-Subaie, M. A. and Chamkha, A. J. Transient natural convection flow of a particulate suspension through a vertical channel. Heat and Mass Transfer. 2004. 40(9): 707-713.

[2] Rajput, U. and Sahu, P. Transient free convection mhd flow between two long vertical parallel plates with constant temperature and variable mass diffusion. Journal of Math. Analysis. 2011. 34(5): 1665-1671.

[3] Singh, A., Gholami, H. R. and Soundalgekar, V. Transient free convection flow between two vertical parallel plates. Heat and Mass Transfer. 1996. 31(5): 329-331.

[4] Narahari, M. Transient free convection flow between long vertical parallel plates with ramped wall temperature at one boundary in the presence of thermal radiation and constant mass diffusion. Meccanica. 2012. 47(8): 1961-1976.

[5] Singh, A. and Sarveshanand. Magnetohydrodynamic free convection between vertical parallel porous plates in the presence of induced magnetic field. SpringerPlus. 2015. 4(1): 333.

[6] Paul, T., Jha, B. and Singh, A. Transient free convective flow in a vertical channel with constant temperature and constant heat flux on walls. Heat and Mass Transfer. 1996. 32(1-2): 61-63.

[7] Rajput, U. and Sahu, P. Combined effects of chemical reactions and heat generation/absorption on unsteady transient free convection mhd flow between two long vertical parallel plates through a porous medium with constant temperature and mass diffusion. Elixir Appl. Math. 2011. 39: 4855-4859.

[8] Sheela-Francisca, J. and Tso, C. Viscous dissipation effects on parallel plates with constant heat flux boundary conditions. International Communications in Heat and Mass Transfer. 2009. 36(3): 249-254.

[9] Kundu, B. Semianalytical methods for heat and fluid flow between two parallel plates. Journal of Thermal Engineering. 2015. 1(3): 175-181.

[10] Yan, W., Tsay, Y. and Lin, T. Effects of wetted walls on laminar natural convection between vertical parallel plates with asymmetric heating. Applied Scientific Research. 1990. 47(1): 45-64.

[11] Lesnic, D., Ingham, D., Pop, I. and Storr, C. Free convection boundary-layer flow above a nearly horizontal surface in a porous medium with Newtonian heating. Heat and Mass Transfer. 2004. 40(9): 665-672.

[12] Shehzad, S., Hayat, T., Alhuthali, M. and Asghar, S. Mhd three-dimensional flow of jeffrey fluid with Newtonian heating. Journal of Central South University. 2014. 21(4): 1428-1433. 
[13] Narahari, M. and Ishak, A. Radiation effects on free convection flow near a moving vertical plate with Newtonian heating. Journal of Applied Sciences. 2011. 11(7): 1096-1104.

[14] Das, M., Mahato, R. and Nandkeolyar, R. Newtonian heating effect on unsteady hydromagnetic casson fluid flow past a flat plate with heat and mass transfer. Alexandria Engineering Journal. 2015. 54(4): 871-879.

[15] Qayyum, S., Hayat, T., Shehzad, S. A. and Alsaedi, A. Effect of a chemical reaction on magnetohydrodynamic (mhd) stagnation point flow of walters-b nanofluid with Newtonian heat and mass conditions. Nuclear Engineering and Technology. 2017. 49(8): 1636-1644.

[16] Jain, P. and Chaudhary, R. Closed form solution of heat and mass transfer past an inclined oscillating surface with Newtonian heating under the effect of thermal radiation and mass diffusion. Adv Appl Sci Res. 2013. 4(6): 285-306.

[17] Hayat, T., Hussain, Z., Farooq, M. and Alsaedi, A. Magnetohydrodynamic flow of powelleyring fluid by a stretching cylinder with Newtonian heating. Thermal Science. 2018. 22(1B): 371-382.

[18] Zin, N. A. M., Khan, I. and Shafie, S. Exact and numerical solutions for unsteady heat and mass transfer problem of Jeffrey fluid with mhd and Newtonian heating effects. Neural Computing and Applications. 2018. 30(11): 3491-3507.

[19] Akbar, N. S. and Nadeem, S. Mixed convective magnetohydrodynamic peristaltic flow of a jeffrey nanofluid with Newtonian heating. Zeitschrift für Naturforschung A. 2013. 68(6-7): 433-441.

[20] Hamza, M. Free convection slip flow of an exothermic fluid in a convectively heated vertical channel. Ain Shams Engineering Journal. 2016. 